\title{
Biochar como tecnología de emisión negativa frente al cambio climático
}

Biochar as a negative emission technology for tackling climate change
Jordy Pariona-Palomino ${ }^{1 *} \oplus$, Wendy Matos-Ormeño ${ }^{2} \odot$
y Eduardo Huillca-Huanaco ${ }^{1 \odot}$
1 Universidad Cientifica del Sur. Lima, Perú.

2 Universidad Nacional San Luis Gonzaga. Ica, Perú.

\section{SOUTH} SUSTAINABILITY

Citar como: Pariona-Palomino, J., Matos-Ormeño, W. y Huillca-Huanaco, E. (2020). «Biochar como tecnología de emisión negativa frente al cambio climático». South Sustainability, 1(2) e014

DOI: $10.21142 /$ SS-0102-2020-014

Artículo recibido: 16/10/2020 Revisado por pares

Artículo aprobado: 14/1/2021

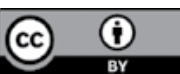

(C) Los autores, 2020. Publicado por la Universidad Científica del Sur (Lima, Perú)

${ }^{*}$ E-mail de correspondencia: 180000101@cientifica.edu.pe

\section{RESUMEN}

El biochar es una alternativa de acción climática, accesible, de bajo costo y sostenible. Su aplicación como enmienda permite incrementar la fertilidad del suelo y reducir las emisiones de gases de efecto invernadero como el $\mathrm{CO}_{2}$. Por ende, es necesario implementar el biochar como tecnología de emisión negativa frente al cambio climático, disminuir los impactos negativos en el ambiente y salvaguardar la salud de las personas. La investigación es una revisión de literatura holística y sistemática disponible sobre el uso del biochar como estrategia de mitigación, debido a su capacidad de secuestro de carbono, y sobre el potencial de sus aplicaciones e interacciones que presenta con el clima, la seguridad alimentaria y los componentes del ecosistema. Se encontró que el potencial de secuestro de carbono presentado por el biochar en equivalencias de $\mathrm{CO}_{2}$ supera las $20 \mathrm{GtCO}_{2}$ eq por año. Esto es muy significativo en la búsqueda de tecnologías de emisiones negativas eficientes que ayuden a reducir las emisiones de $\mathrm{CO}_{2}$ que generan las actividades antropogénicas anualmente.

Palabras clave: sostenibilidad, cambio climático, seguridad alimentaria, biocarbón, emisión negativa

\section{ABSTRACT}

Biochar is an accessible, low-cost and sustainable alternative for climate change action. Its application as a corrective enables increased soil fertility, as well as a reduction in the emission of greenhouse gases such as $\mathrm{CO}_{2}$. Therefore, the implementation of biochar as a negative emission technology is required in order to tackle climate change, reduce negative impacts on the environment, and safeguard human health. This research offers a review of the holistic and systematic literature available on the use of biochar as a mitigation strategy, through its ability to sequester carbon, as well as the potential for its applications and interactions in climate, food security, and ecosystem components. The carbon sequestration potential represented by biochar in $\mathrm{CO}_{2}$ equivalents exceeds $20 \mathrm{GtCO}_{2}$ eq per year, and this is highly significant in the search for efficient negative emission technologies that can help to reduce annual $\mathrm{CO}_{2}$ emissions resulting from anthropogenic activities.

Keyswords: Sustainability, climate change, food security, biochar, negative emission 


\section{Introducción}

El cambio climático se ha convertido en un problema de escala global (Zomeren et al., 2019), principalmente por el aumento de gases de efecto invernadero, como el metano $\left(\mathrm{CH}_{4}\right)$ y el dióxido de carbono $\left(\mathrm{CO}_{2}\right)$. Este último es muy peligroso (Kumari et al., 2018; Zhang y Xu, 2018), ya que está relacionado directamente con la preservación de la vida en el planeta. Las emisiones producidas por las actividades antropogénicas superan los $40 \mathrm{GtCO}_{2}$ por año, de los cuales la agricultura contribuye con el $14 \%$ (Gambhiry Tavoni, 2019; IPCC, 2014). Si no se logra abordar esta situación con acciones climáticas, es probable que se superen los cambios de temperatura de $2{ }^{\circ} \mathrm{C}$ por encima de los niveles preindustriales (Boysen et al., 2017). Ello podría afectar significativamente la salud de la población, los componentes del ecosistema y la economía de todos los países.

El Perú es uno de los países más vulnerables a los efectos del cambio climático (Paun et al., 2018). Se espera que un aumento en la temperatura y una disminución en la precipitación pueda tener efectos negativos en el sector agrícola (Christensen et al., 2013). Por ende, la influencia de la variabilidad climática ha llevado a tomar estrategias de adaptación (Dussi y Flores, 2017), como la diversificación y los cambios a cultivos tolerantes en la región surandina (Ponce, 2020). Además, se ha evaluado que los cambios en la disponibilidad hídrica pueden impactar en los rendimientos del cultivo de maíz (Laudien et al., 2020).

Ante esta problemática, es necesario optar por tecnologías de emisiones negativas (NET) que sean accesibles, eficientes y eviten generar daños para el ambiente (Tisserant y Cherubini, 2019), como la bioenergía con captura y almacenamiento de carbono (Gambhir y Tavoni, 2019), forestación y reforestación, meteorización mejorada (Fawzy et al., 2020) y el biochar (Smith, 2016). Ante ello, el biochar no solo representa una alternativa para aumentar la fertilidad del suelo y el secuestro de carbono (Zimmerman, 2010; Jindo et al., 2014), sino también una estrategia para disminuir los gases de efecto invernadero y evitar la degradación del suelo para mantener la seguridad alimentaria (Gomes et al., 2019).

Se estima que el biochar posee un potencial de secuestro de carbono para generar emisiones negativas de aproximadamente 0,7 GtCeq por año (Smith, 2016). Esta es una alternativa que posee diversas ventajas sobre las demás NET (Li et al., 2019). Así, en equivalencias de captura de $\mathrm{CO}_{2}$ el biochar presenta un potencial entre 2,13 $\mathrm{GtCO}_{2}$ eq y 11,9 $\mathrm{GtCO}_{2}$ eq por año (Lee et al., 2010; Laird et al., 2009; Woolf et al., 2010). Esto ayudaría a reducir las emisiones de tipo antropogénico.

Las aplicaciones del biochar son diversas, pues interactúan con las matrices del ecosistema y el sistema climático de manera positiva. Por ello, la importancia de este trabajo radica en identificar información bibliográfica disponible sobre el biochar y su aplicación como tecnología de emisión negativa frente al cambio climático, por su rol en la sostenibilidad medioambiental, todo ello en concordancia con los objetivos de desarrollo sostenible.

\section{Biochar}

El biochar es un producto abundante en carbono que se obtiene a partir de residuos de biomasa a través de un proceso de degradación térmica en ausencia de oxígeno denominado como pirólisis (Lehmann et al., 2011). Si bien las propiedades fisicoquímicas del biochar varían dependiendo de la materia prima con la que se obtiene (tabla 1), resultan por lo general ser alcalinas, con una gran superficie porosa, cargas variables y varios grupos funcionales (Sun et al., 2018). Por ello, la adición de biochar al suelo puede modificar sus propiedades químicas como los cambios de $\mathrm{pH}$, la conductividad eléctrica (CE), la capacidad de intercambio catiónico (CIC) y niveles de nutrientes (Trakal et al., 2011). Además, pueden estabilizar metales pesados (Beesley y Marmiroli, 2011), mediante interacciones electrostáticas, intercambio iónico y la unión específica de iones metálicos (Wang et al., 2017).

Tabla 1. Propiedades fisicoquímicas del biochar obtenido a partir de diversas materias primas

\begin{tabular}{|c|c|c|c|c|c|c|c|}
\hline $\begin{array}{c}\text { Materia } \\
\text { prima }\end{array}$ & $\begin{array}{c}\mathrm{T} \\
\left({ }^{\circ} \mathrm{C}\right)\end{array}$ & pH & $\begin{array}{c}\mathrm{CIC} \\
(\mathrm{mol} \\
\left.\mathrm{kg}^{-1}\right)\end{array}$ & C (\%) & $\begin{array}{c}\text { Ceniza } \\
(\%)\end{array}$ & $\begin{array}{l}\text { BET } \\
\left(\mathrm{m}^{2}\right. \\
\left.\mathrm{g}^{-1}\right)\end{array}$ & Fuente \\
\hline \multirow{3}{*}{$\begin{array}{l}\text { Madera } \\
\text { de roble }\end{array}$} & 400 & 6,43 & n. d. & 70,52 & n. d. & 5,60 & \multirow{3}{*}{$\begin{array}{l}\text { Jindo } \\
\text { et al., } \\
2014\end{array}$} \\
\hline & 600 & 8,85 & n. d. & 81,22 & n. d. & 288,58 & \\
\hline & 800 & 9,68 & n. d & 82,85 & n. d. & 398,15 & \\
\hline \multirow{3}{*}{$\begin{array}{l}\text { Estiércol } \\
\text { de ave }\end{array}$} & 300 & 8,80 & n. d. & 52,20 & 24,00 & 4,30 & \multirow{3}{*}{$\begin{array}{c}\text { Ahmad } \\
\text { et al., } \\
2014\end{array}$} \\
\hline & 500 & 11,00 & n. d. & 51,56 & 24,00 & 5,80 & \\
\hline & 700 & 10,70 & n. d. & 56,06 & 24,20 & 6,60 & \\
\hline \multirow{4}{*}{$\begin{array}{l}\text { Lodo de } \\
\text { aguas } \\
\text { residuales }\end{array}$} & 300 & 5,32 & n. d. & 25,60 & 52,80 & n. d. & \multirow{4}{*}{$\begin{array}{l}\text { Hossain } \\
\text { et al., } \\
2011\end{array}$} \\
\hline & 400 & 4,87 & n. d. & 20,20 & 63,30 & n. d. & \\
\hline & 500 & 7,27 & n. d. & 20,3 & 68,20 & n. d. & \\
\hline & 700 & 12,00 & n. d. & 20,4 & 72,50 & n. d. & \\
\hline \multirow{2}{*}{$\begin{array}{l}\text { Rastrojo } \\
\text { de maíz }\end{array}$} & 350 & 9,39 & 419,30 & 60,40 & 11,40 & 293 & \multirow{2}{*}{$\begin{array}{l}\text { Nguyen y } \\
\text { Lehmann, } \\
2009\end{array}$} \\
\hline & 600 & 9,42 & 252,10 & 70,60 & 16,70 & 527 & \\
\hline \multirow{3}{*}{$\begin{array}{c}\text { Madera } \\
\text { de } \\
\text { álamo }\end{array}$} & 400 & 9,00 & n. d. & 67,30 & 3,50 & 3,00 & \multirow{3}{*}{$\begin{array}{l}\text { Kloss } \\
\text { et al., } \\
2012\end{array}$} \\
\hline & 460 & 9,20 & n. d. & 70,00 & 5,70 & 8,20 & \\
\hline & 525 & 8,70 & n. d. & 77,90 & 6,80 & 55,7 & \\
\hline
\end{tabular}

Un proceso de pirólisis a alta temperatura $\left(600{ }^{\circ} \mathrm{C}\right)$ genera un biochar con un alto contenido de carbono, lo que determina su carácter recalcitrante. Por el contrario, los que se obtienen a bajas temperaturas $\left(400{ }^{\circ} \mathrm{C}\right.$ ) conservan aún compuestos volátiles (Jindo et al., 2014). El biochar que contenga más carbonos negros aromáticos en su superficie será el más estable en el suelo, a diferencia de cualquier otra forma de carbono orgánico (Sun et al., 


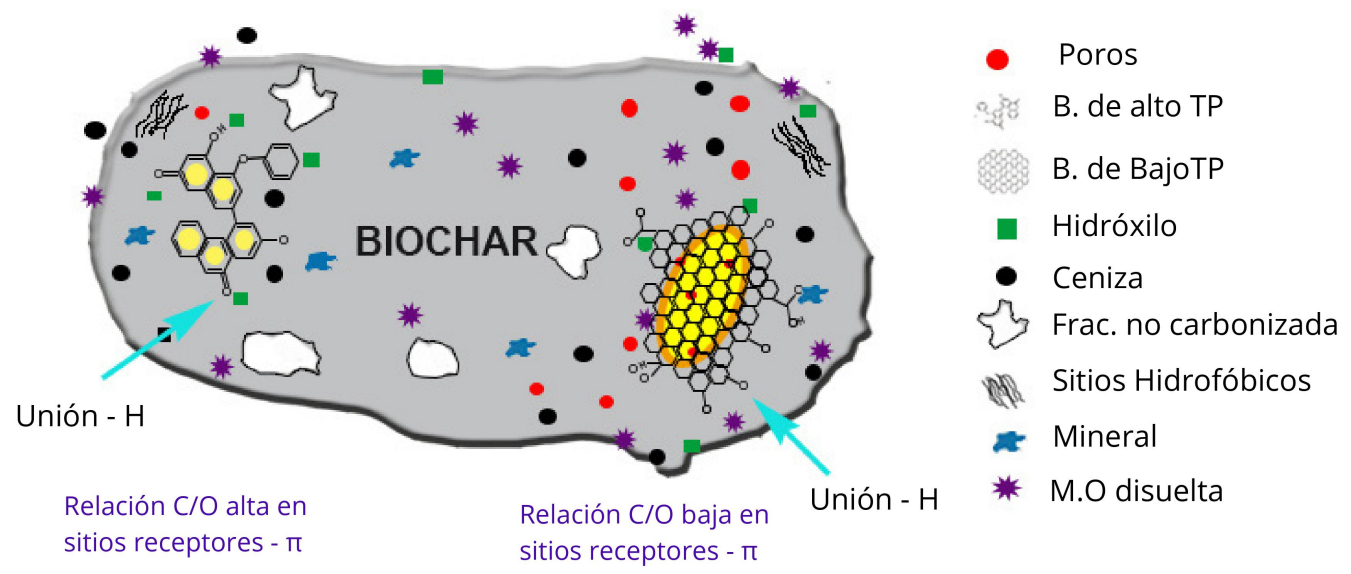

Figura 1. Estructura básica del biochar. Adaptado de Zhang et al., 2018.

2018). Se ha establecido que las tasas de mineralización del biochar son muy bajas, las cuales oscilan entre $10^{2}$ y $10^{7}$ años (Zimmerman, 2010). La estructura del biochar contiene dos fracciones estructurales (figura 1), las películas de grafeno cristalino y las estructuras aromáticas amorfas, ambas enlazadas por el carbono en forma de anillos tipo benceno con oxígeno o hidrógeno, por lo que estos enlaces de C-O y C-H determinan la estabilidad del biochar (Campos et al., 2020; Meng et al., 2021). Por lo tanto, a baja temperatura de pirólisis se obtiene un mayor rendimiento de biochar.

\section{Biochar y cambio climático}

El Acuerdo de París establece que la temperatura debería estar muy por debajo de $2{ }^{\circ} \mathrm{C}$ en 2100 , con la finalidad de minimizar los peligros del cambio climático (Smith, 2016). Sin embargo, para lograr dicho objetivo se debería alcanzar un límite de $1,5{ }^{\circ} \mathrm{C}$ y evitar que las emisiones de $\mathrm{CO}_{2}$ no superen las $320 \mathrm{GtCO}_{2}$ (Holz et al., 2018). Si bien las emisiones producidas por las actividades antropogénicas superan las $40 \mathrm{GtCO}_{2}$ por año (Gambhir y Tavoni, 2019), a ese paso, evidentemente, si no se toman acciones drásticas, podríamos superar dicho límite. Dichas medidas van desde la captura de carbono en el aire conocidas como DACCS, que implican un secuestro directo (Gambhir y Tavoni, 2019); forestación intensiva (Griscom et al., 2017); y hasta la aplicación de tecnologías de emisiones negativas (NET) (Tisserant y Cherubini, 2019), las cuales se han venido usando con el propósito de mitigación, cada una con un impacto significativo en el ambiente (tabla 2).

El informe de IPCC (2018) sugiere incluso la implementación y aplicación de estas tecnologías para capturar el $\mathrm{CO}_{2}$ presente en la atmósfera; asimismo, se aprecia una mayor efectividad de las tecnologías a partir de 2050 (figura 2). Por un lado, en el escenario A1 no se incluyen las BECCS, por lo que se prevé un escenario social en donde la forestación es la fuente de captura de $\mathrm{CO}_{2}$; por
Tabla 2. Comparación de impactos entre diversas tecnologías de emisión negativa

\begin{tabular}{|c|c|c|c|c|c|}
\hline NET & 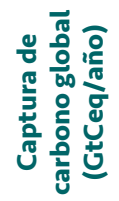 & 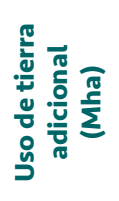 & 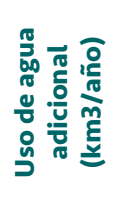 & 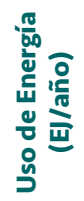 & Fuente \\
\hline BECCS & 3,3 & 380 & 720 & -170 & $\begin{array}{c}\text { Smith et al., } \\
2015\end{array}$ \\
\hline DAC & 3,3 & ND & 10 & 156 & $\begin{array}{c}\text { McLaren, } \\
2012\end{array}$ \\
\hline Biochar & 1,3 & 0 & 0 & -14 & Smith, 2016 \\
\hline Reforestación & 1,5 & 320 & 370 & ND & $\begin{array}{l}\text { McLaren, 2012 } \\
\text { Smith et al., } \\
2015\end{array}$ \\
\hline $\begin{array}{l}\text { Secuestro } \\
\text { de carbono } \\
\text { en el suelo }\end{array}$ & 1,2 & 0 & 0 & 0 & Lal, 2004 \\
\hline
\end{tabular}

otro lado, en el escenario A4 se muestra un panorama de consumo intensivo de recursos donde se generan grandes cantidades de $\mathrm{CO}_{2}$ y las BECCS son el único medio para reducir las emisiones.

El biochar es conocido como una de las NET de bajo costo para el secuestro de $\mathrm{CO}_{2}$. Además, se puede emplear como enmienda para la agricultura, lo que contribuye a la mitigación del cambio climático (Fawzy et al., 2020; Smith, 2016). Gracias al poder recalcitrante (es decir, resistentes a la degradación química y biológica), se convierte en un sumidero potencial de carbono (Cheng et al., 2008). Y debido a que una parte significativa de este no se descompone, el biochar evita que el carbono de la biomasa retorne a la atmósfera como $\mathrm{CO}_{2}$, y permanezca capturado en el suelo durante largos periodos (Mohan et al., 2018). Asimismo, el biochar posee un potencial de secuestro de carbono para generar emisiones negativas 


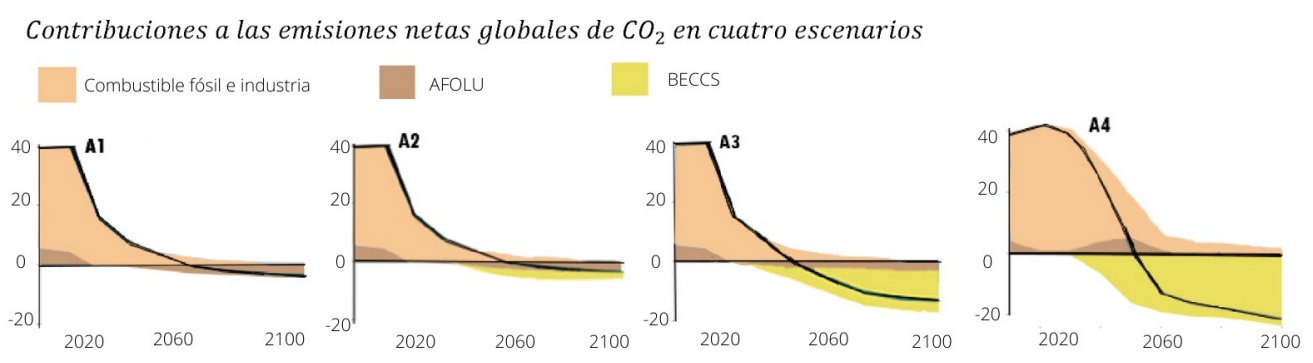

Figura 2. Contribuciones de las remociones de $\mathrm{CO}_{2}$ de la agricultura y otros usos de suelo (AFOLU), bioenergía con captura de $\mathrm{CO}_{2}$ (BECCS), tecnologías de emisiones negativas. Adaptado de IPCC, 2018.

de aproximadamente 0,7 GtCeq por año (Smith, 2016). Por esta razón, el secuestro de carbono por parte del biochar es una alternativa que posee diversas ventajas sobre las demás NET en relación con los impactos que estos ocasionan (Smith, 2016; Li et al., 2019). En términos de captura de $\mathrm{CO}_{2}$, el biochar presenta un potencial que varía entre $2,13 \mathrm{GtCO}_{2}$ eq y $11,9 \mathrm{GtCO}_{2}$ eq por año (Lee et al., 2010; Laird et al., 2009; Powel y Lenton, 2012; Woolf et al., 2010). Si esto se intensifica, podría ayudar a minimizar las emisiones producidas por el hombre. En la figura 3 se muestra cómo funciona el biochar como una NET eficiente. $\mathrm{El} \mathrm{CO}_{2}$ atmosférico es captado por las plantas, los residuos de estas pasan por un proceso de pirólisis y generan subproductos del biochar, los cuales tienen múltiples aplicaciones que emiten $\mathrm{CO}_{2}$. No obstante, parte del $\mathrm{CO}_{2}$ es capturado por el biochar y no lo libera al ambiente, por lo que convierte en una tecnología de emisión negativa que aporta a la mitigación del cambio climático.

\section{Biochar y agroecología}

El biochar incorpora diversos beneficios al sistema suelo (Ferjani et al., 2020). Diferentes trabajos mencionan que la aplicación de biocarbón trae efectos positivos, como el mejoramiento de los suelos meteorizados, ácidos, con bajo CIC y también aumenta el porcentaje de humedad del suelo (Tisserant y Cherubini, 2019; Lorenz y Lal, 2014). Asimismo, tiene la capacidad de inmovilizar plaguicidas y brinda a las plantas una mejor respuesta frente a patógenos (Baharum et al., 2020; Varjani et al., 2019; Sanchez-Hernandez et al., 2019). Además, el uso de biocarbón ayuda a disminuir el estrés que presentan las plantas al encontrarse en suelos salinos (Sun et al., 2019; Nguyen et al., 2018; Saifullah et al., 2018).

Durante los últimos años, la aplicación de compuestos agroquímicos ha ocasionado la acidificación de las tierras

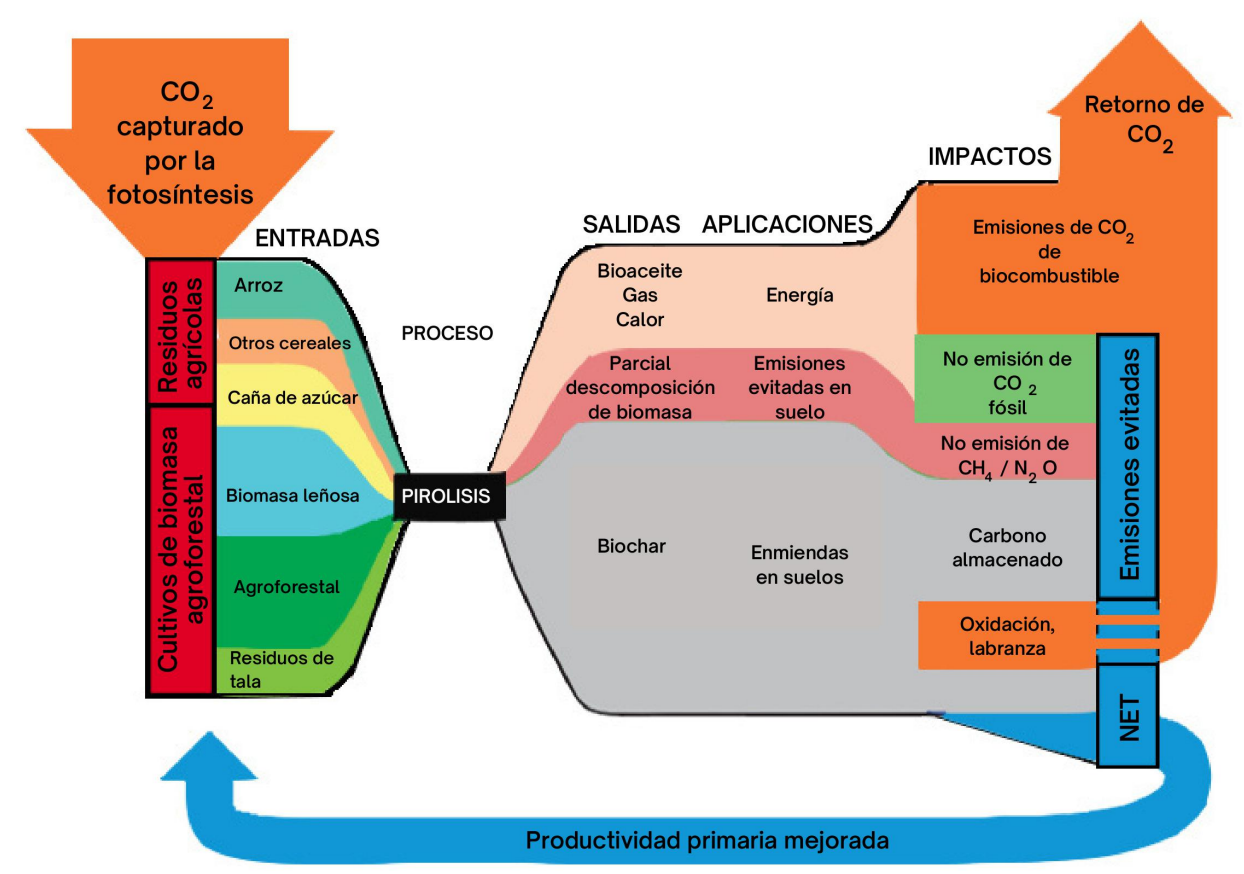

Figura 3. Biochar como tecnología de emisión negativa. Adaptado de Woolf et al., 2010. 
de cultivo, baja disponibilidad de nutrientes, problemas de toxicidad en el suelo, entre otros (Carvalho, 2017; Rickson et al., 2015). En estos casos el biochar juega un papel de agente encalado para evitar la degradación del suelo (Fidel et al., 2017; Tisserant y Cherubini, 2019).

Debido a que la erosión del suelo es un peligro para la seguridad alimentaria (Arora et al., 2018; Deng, et al., 2018; Poesen, 2018; Altieri y Nicholls, 2013), urge tomar medidas, ya que se sostiene (Han et al., 2020; Abd-Elmabod et al., 2020; Asfaw et al., 2020) que en suelos muy erosionados el rendimiento agrícola puede disminuir entre $65 \%$ y $80 \%$. Ante ello, el biochar disminuye el riesgo de degradación de los suelos, que están relacionados indirectamente para garantizar la seguridad alimentaria. Por esta razón, las acciones que garantizan la seguridad alimentaria deberían abarcar políticas en torno al desarrollo de la producción agrícola sostenible y resiliente (Sartori et al., 2019; Deng, et al., 2018; Gomes et al., 2019).

Por esto, la agroecología desempeña una función importante que engloba principios de la ecología y sociología, entre otros (Dussi y Flores, 2017; Van der Ploeg et al., 2019; Gliessman, 2016; Nair et al., 2017), para manejar y evaluar la gestión de una agricultura sostenible, al aportar ventajas para la sociedad en términos económicos y medioambientales (Ramírez-Iglesias et al., 2017; Wezel et al., 2016; Mier et al., 2018).

Por ende, dos de los principios básicos de esa ciencia: optimizar la disponibilidad de nutrientes y garantizar que las condiciones del sistema suelo sean favorables para el desarrollo de las plantas (Snapp, 2017; Aguilera et al., 2020; Hale et al., 2020), podrían estar relacionados directamente con la aplicación del biochar, pues este cumple dichas funciones y evita así la degradación del suelo. Además, diferentes estudios (Mohan et al., 2018; Hale et al., 2020; Saletnik et al., 2018; Ferjani et al., 2020) mencionan que durante el proceso de producción del biochar se generan cenizas, que contienen los micronutrientes que el suelo necesita y evitan la quema de residuos agrícolas. Asimismo, hacen de este un método de captura de carbono en la agricultura, que mejora el rendimiento agrícola, el secuestro de carbono y, por ende, la mitigación frente al cambio climático.

\section{Desafíos en su implementación}

Si bien los beneficios del biochar son sustancialmente grandes en términos de mitigación al cambio climático, se deben tener en cuenta ciertas consideraciones para su producción y algunos aspectos o interacciones que presenta con el sistema suelo. Esta revisión ha mostrado diversos beneficios del biochar (Nair et al., 2017). No obstante, el biochar, al interactuar con el suelo, también presenta algunos riesgos, ya que posee la característica de recalcitrante y, por ello, una parte de dicho carbón puede oxidarse y modificar algunas propiedades fisicoquímicas del suelo (Cheng et al., 2008).
En cuanto a la producción de biochar, los estudios dan cuenta de que el emplear cultivos específicos para su producción podría conllevar a la competencia por el uso de suelo y pondría en riesgo a la seguridad alimentaria y degradación ambiental (Boysen et al., 2017). Asimismo, múltiples materias primas podrían contener compuestos tóxicos por bioacumulación, lo que afectaría al crecimiento de las plantas (Qiu et al., 2015; Hilber et al., 2017). Ello requiere una caracterización completa antes de emplearlo.

Por un lado, se da cuenta de que el biochar tiene gran potencial de remoción de metales pesados en los suelos (Jun et al., 2020; Beesley y Marmiroli, 2011). Sin embargo, en los casos de plaguicidas reduce su biodegradación y podría afectar a organismos no objetivos (Khalid et al., 2020). Por otro lado, existe poca literatura sobre las emisiones de carbón negro (Braun et al., 2020) y de cuál es la interacción final del biochar con los sistemas de agua dulce (Tisserant y Cherubini, 2019), lo que podría implicar limitaciones para la mitigación del cambio climático y el riesgo de toxicidad respectivamente.

Es necesario que los países implementen políticas públicas de gestión, ya que el suministro para la producción de biochar solo se centra en el uso de residuos forestales y agrícolas (Fawzy et al., 2020), lo que pondría una limitación importante para el desarrollo y avance de esta tecnología de emisión negativa, que es sumamente importante para la mitigación del $\mathrm{CO}_{2}$ generado por las actividades antropogénicas (Gambhir y Tavoni, 2019). Asimismo, estas políticas deben garantizar disponibilidad de alimentos y evitar la deforestación.

\section{Conclusiones}

El uso del biochar como estrategia para la mitigación frente al cambio climático ha demostrado ser una tecnología exitosa y sostenible. A diferencia de otras tecnologías de emisiones negativas, esta produce los menores impactos en el ambiente. El uso del biochar ayudará a la reducción de $\mathrm{CO}_{2}$ y con ello se lograría cumplir lo planteado por el Acuerdo de París, mantener la temperatura de $1,5^{\circ} \mathrm{C}$ en comparación con los niveles preindustriales.

Esta revisión ha mostrado que el biochar también mejora la calidad de los suelos e incluso ayuda al incremento de la producción agrícola. Estos aspectos son fundamentales para garantizar la seguridad alimentaria $y$, a la vez, minimizar los impactos negativos del cambio climático que cada vez se sienten más. Ejemplos de ello son las sequías, las olas de calor, la menor disponibilidad de agua en acuíferos, entre otros.

El potencial de secuestro de carbono que presenta el biochar en equivalencias de $\mathrm{CO}_{2}$ supera las $20 \mathrm{GtCO}_{2} \mathrm{eq}$ por año, algo muy significativo en la búsqueda de tecnologías de emisiones negativas eficientes que ayuden a reducir las grandes cantidades de $\mathrm{GtCO}_{2}$ emitidas por las 
actividades antropogénicas anualmente. Si no realizamos acciones climáticas para mitigar estos problemas, nos veríamos expuestos a un incremento exponencial de la concentración de $\mathrm{CO}_{2}$ en el ambiente.

El criterio que se debe aplicar para la producción del biochar obtenida a partir de la biomasa agrícola u otros restos vegetales es que estos se cultiven o se aprovechen a un ritmo sostenible, sin haberse expuesto el suelo a una degradación. Se podrían emplear las tierras abandonadas u otros. Además, es necesario diseñar nueva tecnología para la elaboración del biochar al eliminar el hollín y así recuperar parte de la energía del proceso.

En la agricultura, la resiliencia a los fenómenos climáticos está íntimamente relacionada con la biodiversidad y los sistemas agrícolas tradicionales. Bajo ese escenario, aplicar los principios estratégicos de la agroecología, como el eficiente manejo del suelo, es un camino viable para incrementar la sostenibilidad, el crecimiento económico, la productividad agrícola y la mitigación del cambio climático.

\section{Agradecimiento}

El presente manuscrito se publica como parte del programa del curso taller «Publicando en revistas indizadas año 2020», organizado por la Dirección General de Investigación, Desarrollo e Innovación de la Universidad Científica del Sur, en el que participó el investigador principal.

\section{Fuente de financiamiento}

Este trabajo fue autofinanciado.

\section{Contribución de autoría}

JP-P, investigador principal, se encargó del diseño del trabajo y la elaboración del manuscrito. WM-O se encargó del diseño del trabajo, la redacción y el soporte técnico. $\mathrm{EH}-\mathrm{H}$, asesor principal, fue responsable del diseño del trabajo, la redacción y el soporte metodológico. Todos los autores aprobaron la versión final del manuscrito.

\section{Potenciales conflictos de interés}

Los autores declaran que no existe ningún conflicto de interés.

\section{Referencias bibliográficas}

Abd-Elmabod, S., Muñoz-Rojas, M., Jordán, A., Anaya-Romero, M., Phillips, J., Jones, L., Zhang, Z., Pereira, P., Fleskens, L., Van der Ploeg, M. y De la Rosa, D. (2020). «Climate change impacts on agricultural suitability and yield reduction in a Mediterranean region». Geoderma, 374. DOI: 10.1016/j.geoderma.2020.114453

Aguilera, E., Díaz-Gaona, C., García-Laureano, R., Reyes-Palomo, C., Guzmán, G. I., Ortolani, L., Sánchez Rodríguez, M. y RodríguezEstévez, V. (2020). «Agroecology for adaptation to climate change and resource depletion in the Mediterranean region. A review». Agricultural Systems, 181, pp. 1-21. DOI: 10.1016/j.agsy.2020.102809.
Ahmad, M., Rajapaksha, A. U., Lim, J. E., Zhang, M., Bolan, N., Mohan, D., Vithanage, M., Lee, S. y Ok, Y. S. (2014). «Biochar as a sorbent for contaminant management in soil and water: A review». Chemosphere, 99, pp. 19-33. DOI: 10.1016/j.chemosphere.2013.10.071

Altieri, M. A. y Nicholls, C. I. (2013). «The adaptation and mitigation potential of traditional agriculture in a changing climate». Climatic Change, 140(1), pp. 33-45. DOI: 10.1007/s10584-013-0909-y.

Arora, N. K., Fatima, T., Mishra, I., Verma, M., Mishra, J. y Mishra, V. (2018). «Environmental sustainability: challenges and viable solutions». Environmental Sustainability, 1, pp. 309-340. DOI: 10.1007/s42398-018-00038-w.

Asfaw, S., Pallante, G. y Palma, A. (2020). «Distributional impacts of soil erosion on agricultural productivity and welfare in Malawi». Ecological Economics, 177. DOI: 10.1016/j.ecolecon.2020.106764.

Baharum, N., Nasir, H. M., Ishak, M., Isa, N., Hassan, M. y Aris, A. (2020). «Highly efficient removal of diazinon pesticide from aqueous solutions by using coconut shellmodified biochar». Arabian Journal of Chemistry, 13(7), pp. 6106-6121. DOI: 10.1016/j.arabjc.2020.05.011.

Beesley, L. y Marmiroli, M. (2011). «The immobilisation and retention of soluble arsenic, cadmium and zinc by biochar». Environmental Pollution, 159(2), pp. 474-480. DOI: 10.1016/j.envpol.2010.10.016.

Boysen, L. R., Lucht, W., Gerten, D., Heck, V., Lenton, T. M. y Schellnhuber, H. J. (2017). "The limits to global-warming mitigation by terrestrial carbon removal». Earth's Future, 5(5), pp. 463-474. DOI: 10.1002/2016ef000469.

Braun, M., Kappenberg, A., Sandhage-Hofmann, A. y Lehndorff, E. (2020). «Leachable soil black carbon after biochar application». Organic Geochemistry, 143. DOI: 10.1016/j.orggeochem.2020.103996.

Campos, P., Miller, A., Knicker, H., Costa-Pereira, M., Merino, A. y De la Rosa, J. (2020). «Chemical, physical and morphological properties of biochars produced from agricultural residues: Implications for their use as soil amendment». Waste Management, 105, pp. 256-267. DOI: 10.1016/j.wasman.2020.02.013.

Carvalho, F. P. (2017). «Pesticides, environment, and food safety». Food and Energy Security, 6(2), pp. 48-60. DOI: 10.1002/fes3.108.

Cheng, C.-H., Lehmann, J. y Engelhard, M. H. (2008). «Natural oxidation of black carbon in soils: Changes in molecular form and surface charge along a climosequence». Geochimica et Cosmochimica Acta, 72(6), pp. 1598-1610. DOI: 10.1016/j.gca.2008.01.010.

Christensen, J. H., Krishna Kumar, K., Aldrian, E., An, S., Cavalcanti, I., De Castro, M., Dong, W., Goswami, P., Hall, A., Kanyanga, J., Kitoh, A., Kossin, J. y Zhou, T. (2013). «Climate phenomena and their relevance for future regional climate change». En: Stocker, T. F., Qin, D., Plattner, G.-K., Tignor, M., Allen, S. K., Boschung, J., Nauels, A., Xia, Y., Bex, V. y Midgley, P. M. (Eds.), Climate Change 2013: The Physical Science Basis. Contribution of Working Group I to the Fifth Assessment Report of the Intergovernmental Panel on Climate Change. Cambridge y Nueva York: Cambridge University Press. DOI: 10.1017/CBO9781107415324.028.

Deng, X., Xu, D., Zeng, M. y Qi, Y. (2018). «Landslides and cropland abandonment in China's mountainous areas: Spatial distribution, empirical analysis and policy implications». Sustainability, 10(11), pp. 3909-3925. DOI: 10.3390/su10113909.

Dussi, M. C. y Flores, L. B. (2017). «Visión multidimensional de la agroecología ante el cambio climático». Interdisciplina, 6(14), pp. 129-153. DOI: 10.22201/ceiich.24485705e.2018.14.63384.

Fawzy, S., Osman, Al., Doran, J. y Rooney, D. W. (2020). «Estrategias para la mitigación del cambio climático: una revisión». Environ Chem Lett, pp. 1-26. DOI: 10.1007/s10311-020-01059-w. 
Ferjani, A., Jellali, S., Akrout, H., Limousy, L., Hamdi, H., Thevenin, N. y Jeguirim, M. (2020). «Nutrient retention and release from raw exhausted grape marc biochars and an amended agricultural soil: Static and dynamic investigation». Environmental Technology \& Innovation, 19. DOI: 10.1016/j.eti.2020.100885.

Fidel, R. B., Laird, D. A., Thompson, M. L. y Lawrinenko, M. (2017). «Characterization and quantification of biochar alkalinity». Chemosphere, 167, pp. 367-373. DOI: 10.1016/j. chemosphere.2016.09.151.

Gambhir, A. y Tavoni, M. (2019). «Direct air carbon capture and sequestration: How it works and how it could contribute to climatechange mitigation». One Earth, 1(4), pp. 405-409. DOI: 10.1016/j. oneear.2019.11.006.

Gliessman, S. (2016). «Transforming food systems with agroecology». Journal of Rural Studies, 71, pp. 187-189. DOI: 10.1080/21683565.2015.1130765.

Gomes, L., Simões, S., Nora, E., De Sousa-Neto, E., Forti, M. y Ometto, J. (2019). «Agricultural expansion in the Brazilian Cerrado: Increased soil and nutrient losses and decreased agricultural productivity». Land, 8(1), pp. 12. DOI: 10.3390/land8010012.

Griscom, B. W., Adams, J., Ellis, P. W., Houghton, R. A., Lomax, G., Miteva, D. A. et al. (2017). «Natural climate solutions». Proceedings of the National Academy of Sciences, 114(44), pp. 11645-11650. DOI: 10.1073/pnas.1710465114

Hale, S. E., Nurida, N. L., Jubaedah, Mulder, J., Sørmo, E., Silvani, L., Abiven, S., Joseph, S., Taherymoosavi, S. y Cornelissen, G. (2020). «The effect of biochar, lime and ash on maize yield in a longterm field trial in a Ultisol in the humid tropics». Science of The Total Environment, 719. DOI: 10.1016/j.scitotenv.2020.137455.

Han, J., Ge, W., Hei, Z., Cong, C., Ma, C., Xie, M., Liu, B., Feng, W., Wang, F. y Jiao, J. (2020). «Agricultural land use and management weaken the soil erosion induced by extreme rainstorms». Agriculture, Ecosystems \& Environment, 301. DOI: 10.1016/j.agee.2020.107047.

Hilber, I., Bastos, A. C., Loureiro, S., Soja, G., Marsz, A., Cornelissen, G. y Bucheli, T. D. (2017). "The different faces of biochar: Contamination risk versus remediation tool». Journal of Environmental Engineering and Landscape Management, 25(2), pp. 86-104. DOI: 10.3846/16486897.2016.1254089.

Holz, C., Siegel, L. S., Johnston, E., Jones, A. P. y Sterman, J. (2018). «Ratcheting ambition to limit warming to $1.5^{\circ} \mathrm{C}$ - trade-offs between emission reductions and carbon dioxide removal». Environmental Research Letters, 13(6), pp. 064028. DOI: 10.1088/1748-9326/aac0c1.

Hossain, M. K., Strezov, V., Chan, K. Y., Ziolkowski, A. y Nelson, P. F. (2011). «Influence of pyrolysis temperature on production and nutrient properties of wastewater sludge biochar». Journal of Environmental Management, 92(1), pp. 223-228. DOI: 10.1016/j. jenvman.2010.09.008.

Intergovernmental Panel on Climate Change (IPCC) (2014). «Cambio climático 2014». En: Pachauri, R. y Meyer, L. (Eds.), Informe de síntesis sobre el cambio climático (p. 49). Ginebra: IPCC.

Intergovernmental Panel on Climate Change (IPCC) (2018). «Resumen para responsables de políticas». En: Mansson-Delmotte, V., Zhay, H., Portner, H., Roberts, D. Skea, J., Sukla, P., et al. (Eds.), Calentamiento global de $1.5^{\circ} \mathrm{C}$, Informe especial del IPCC (p. 16). Ginebra: IPCC.

Jindo, K., Mizumoto, H., Sawada, Y., Sanchez-Monedero, M. A. y Sonoki, T. (2014). «Physical and chemical characterization of biochars derived from different agricultural residues». Biogeosciences, 11(23), pp. 6613-6621. DOI: 10.5194/bg-11-6613-2014.

Jun, L., Wei, H., Aili, M., Juan, N., Hongyan, X., Jingsong, H., Yunhua, Z. y Cuiying, P. (2020). «Effect of lychee biochar on the remediation of heavy metal-contaminated soil using sunflower: a field experiment». Environmental Research, 188. DOI: 10.1016/j. envres.2020.109886.
Khalid, S., Shahid, M., Murtaza, B., Bibi, I., Natasha, Asif Naeem, M. y Khan Niazi, N. (2019). «A critical review of different factors governing the fate of pesticides in soil under biochar application». Science of The Total Environment, 711. DOI: 10.1016/j. scitotenv.2019.134645.

Kloss, S., Zehetner, F., Dellantonio, A., Hamid, R., Ottner, F., Liedtke, V., Schwanninger, M., Gerzabek, M. y Soja, G. (2012). «Characterization of slow pyrolysis biochars: Effects of feedstocks and pyrolysis temperature on biochar properties». Journal of Environment Quality, 41(4), pp. 990. DOI: 10.2134/jeq2011.0070.

Kumari, S., Hiloidhari, M., Kumari, N., Naik, S. N. y Dahiya, R. P. (2018). «Climate change impact of livestock CH4 emission in India: Global temperature change potential (GTP) and surface temperature response». Ecotoxicology and Environmental Safety, 147, pp. 516-522. DOI: 10.1016/j.ecoenv.2017.09.003.

Laird, D. A., Brown, R. C., Amonette, J. E. y Lehmann, J. (2009). «Review of the pyrolysis platform for coproducing bio-oil and biochar». Biofuels, Bioproducts and Biorefining, 3(5), pp. 547-562. DOI: $10.1002 /$ bbb.169.

Lal, R. (2004). «Soil carbon sequestration impacts on global climate change and food security». Science, 304(5677), pp. 1623-1627. DOI: 10.1126/science.1097396.

Laudien, R., Schauberger, B., Gleixner, S. y Gornoft, C. (2020). «Assessment of weather-yield relations of starchy maize at different scales in Peru to support the NDC implementation». Agricultural and Forest Meteorology, 295. DOI: 10.1016/j.agrformet.2020.108154.

Lee, J. W., Hawkins, B., Day, D. M. y Reicosky, D. C. (2010). «Sustainability: the capacity of smokeless biomass pyrolysis for energy production, global carbon capture and sequestration». Energy \& Environmental Science, 3(11), pp. 1695. DOI: 10.1039/ c004561f.

Lehmann, J., Rillig, M. C., Thies, J., Masiello, C. A., Hockaday, W. C. y Crowley, D. (2011). «Biochar effects on soil biota - A review». Soil Biology and Biochemistry, 43(9), pp. 1812-1836. DOI: 10.1016/j. soilbio.2011.04.022.

Li, L., Yao., You, S., Wang, C., Chong. y Wang, X. (2019). «Optimal design of negative emission hybrid renewable energy systems with biochar production». Applied Energy, 243, pp. 233-249. DOI: 10.1016/j. apenergy.2019.03.183.

Lorenz, K. y Lal, R. (2014). «Biochar application to soil for climate change mitigation by soil organic carbon sequestration». Journal of Plant Nutrition and Soil Science, 177(5), pp. 651-670. DOI: 10.1002/ jpln.201400058.

McLaren, D. (2012). «A comparative global assessment of potential negative emissions technologies». Process Safety and Environmental Protection, 90(6), pp. 489-500. DOI: 10.1016/j.psep.2012.10.005.

Meng, F., Wang, D. y Zhang. (2021). «Effect of ultrasonic vibration-assisted pelleting of biomass on biochar properties». Journal of Cleaner Production, 279, p. 123900. DOI: 10.1016/j. jclepro.2020.123900.

Mier, M., Cacho, T., Giraldo, O. F., Aldasoro, M., Morales, H., Ferguson, B. G., Rosset, P., Khadse, A. y Campos, C. (2018). «Bringing agroecology to scale: key drivers and emblematic cases». Agroecology and Sustainable Food Systems, 42(6), pp. 637-665. DOI: $10.1080 / 21683565.2018 .1443313$.

Mohan, D., Abhishek, K., Sarswat, A., Patel, M., Singh, P. y Pittman, C. U. (2018). «Biochar production and applications in soil fertility and carbon sequestration - a sustainable solution to crop-residue burning in India». RSC Advances, 8(1), pp. 508-520. DOI: 10.1039/ c7ra10353k. 
Nair, V., Nair, P., Dari, B., Freitas, A., Chatterjee, N. y Pinheiro, F. (2017). «Biochar in the Agroecosystem Climate Change Sustainability Nexus». Frontiers in Plant Science, 8, pp. 2051. DOI: 10.3389/fpls.2017.02051.

Nguyen, B. T. y Lehmann, J. (2009). «Black carbon decomposition under varying water regimes». Organic Geochemistry, 40(8), pp. 846-853. DOI: 10.1016/j.orggeochem.2009.05.004.

Nguyen, B. T., Trinh, N., Le, C., Nguyen, T., Tran, T. V., Thai, B. V. y Le, T. (2018). «The interactive effects of biochar and cow manure on rice growth and selected properties of salt-affected soil». Archives of Agronomy and Soil Science, 64(12), pp. 1744-1758. DOI: 10.1080/03650340.2018.1455186.

Paun, A., Acton, L. y Chan, W. S. (2018). «Fragile planet: Scoring climate risks around the world». Londres: HSBC Bank Plc.

Poesen, J. (2018). «Soil erosion in the Anthropocene: Research needs». Earth Surface Processes and Landforms, 43(1), pp. 64-84. DOI: 10.1002/esp.4250.

Ponce, C. (2020). «Intra-seasonal climate variability and crop diversification strategies in the Peruvian Andes: A word of caution on the sustainability of adaptation to climate change». World Development, 127, pp. 104740. DOI: 10.1016/j.worlddev.2019.104740.

Powell, T. W. R. y Lenton, T. M. (2012). «Future carbon dioxide removal via biomass energy constrained by agricultural efficiency and dietary trends». Energy \& Environmental Science, 5(8), pp. 8116. DOI: 10.1039/ c2ee21592f.

Qiu, M., Sun, K., Jin, J., Han, L., Sun, H., Zhao, Y., Xia, X., Wu, F. y Xing, B. (2015). «Metal/metalloid elements and polycyclic aromatic hydrocarbon in various biochars: The effect of feedstock, temperature, minerals, and properties». Environmental Pollution, 206, pp. 298-305. DOI: 10.1016/j.envpol.2015.07.026

Ramírez-Iglesias, E., Hernández-Hernández, R. M., Castro, I. y González, I. (2017). «Management of local organic resources, as an agroecological strategy for the development of fertilizers, in cloud forests of the coastal mountain range-Venezuela». Agro Sur, 45(1), pp. 19-30. DOI: 10.4206/agrosur.2017.v45n1-04.

Rickson, R. J., Deeks, L. K., Graves, A., Harris, J. A. H., Kibblewhite, M. G. y Sakrabani, R. (2015). «Input constraints to food production: the impact of soil degradation». Food Security, 7(2), pp. 351-364. DOI: 10.1007/s12571-015-0437-x.

Saifullah, M., Dahlawi, S., Naeem, A., Rengel, Z. y Naidu, R. (2018). «Biochar application for the remediation of salt-affected soils: Challenges and opportunities». Science of The Total Environment, 625, pp. 320-335. DOI: 10.1016/j.scitotenv.2017.12.257.

Saletnik, B., Zagula, C., Bajcar, M., Czernicka, M. y Puchalski, C. (2018). «Biochar and biomass ash as a soil ameliorant: The effect on selected soil properties and yield of giant miscanthus (Miscanthus $x$ giganteus)». Energies, 11, pp. 2535. DOI: 10.3390/en11102535.

Sanchez-Hernandez, J. C., Ro, K. S. y Díaz, F. J. (2019). «Biochar and earthworms working in tandem: Research opportunities for soil bioremediation». Science of The Total Environment, 688, pp. 574-583. DOI: 10.1016/j.scitotenv.2019.06.212.

Sartori, M., Philippidis, G., Ferrari, E., Borrelli, P., Lugato, E., Montanarella, L. y Panagos, P. (2019). "A linkage between the biophysical and the economic: Assessing the global market impacts of soil erosion». Land Use Policy, 86, pp. 299-312. DOI: 10.1016/j. landusepol.2019.05.014.

Smith, P. (2016). «Soil carbon sequestration and biochar as negative emission technologies». Clobal Change Biology, 22(3), pp. 1315-1324. DOI: $10.1111 / g c b .13178$.
Smith, P., Davis, S. J., Creutzig, F., Fuss, S., Minx, J., Gabrielle, B. et al. (2015). «Biophysical and economic limits to negative $\mathrm{CO} 2$ emissions». Nature Climate Change, 6(1), pp. 42-50. DOI: 10.1038/ nclimate2870.

Snapp, S. (2017). «Agroecology: Principles and practice». Agricultural Systems, pp. 33-72. DOI:10.1016/b978-0-12-802070-8.00002-5.

Sun, H., Shi, W., Zhou, M., Ma, X. y Zhang, H. (2019). «Effect of biochar on nitrogen use efficiency, grain yield and amino acid content of wheat cultivated on saline soil». Plant, Soil and Environment, 65, pp. 83-89. DOI: 10.17221/525/2018-pse.

Sun, W., Zhang, S. y Su, C. (2018). «Impact of biochar on the bioremediation and phytoremediation of heavy metal(loid)s in soil». Advances in Bioremediation and Phytoremediation, pp. 150-169. DOI: 10.5772/intechopen.70349.

Tisserant, A. y Cherubini, F. (2019). «Potentials, limitations, cobenefits, and trade-offs of biochar applications to soils for climate change mitigation». Land, 8(12), pp. 179. DOI: 10.3390/land8120179.

Trakal, L., Komárek, M., Száková, J., Zemanová, V. y Tlustoš, P. (2011). «Biochar application to metal-contaminated soil: Evaluating of Cd, $\mathrm{Cu}, \mathrm{Pb}$ and $\mathrm{Zn}$ sorption behavior using single- and multi-element sorption experiment». Plant, Soil and Environment, 57(8), pp. 372380. DOI: 10.17221/155/2011-pse.

Van der Ploeg, J. D., Barjolle, D., Bruil, J., Brunori, G., Madureira, L. M., Dessein, J., et al. (2019). «The economic potential of agroecology: Empirical evidence from Europe». Journal of Rural Studies, 71, pp. 4661. DOI: 10.1016/j.jrurstud.2019.09.003.

Varjani, S., Kumar, G. y Rene, E. R. (2019). «Developments in biochar application for pesticide remediation: Current knowledge and future research directions». Journal of Environmental Management, 232, pp. 505-513. DOI: 10.1016/j.jenvman.2018.11.043.

Wang, T., Sun, H., Ren, X., Li, B. y Mao, H. (2017). «Evaluation of biochars from different stock materials as carriers of bacterial strain for remediation of heavy metal-contaminated soil». Scientific Reports, 7(1), pp. 1-10. DOI: 10.1038/s41598-017-12503-3.

Wezel, A., Brives, H., Casagrande, M., Clément, C., Dufour, A. y Vandenbroucke, P. (2016). «Agroecology territories: places for sustainable agricultural and food systems and biodiversity conservation». Agroecology and Sustainable Food Systems, 40(2), pp. 132-144. DOI: 10.1080/21683565.2015.1115799.

Woolf, D., Amonette, J. E., Street-Perrott, F. A., Lehmann, J. y Joseph, S. (2010). «Sustainable biochar to mitigate global climate change». Nature Communications, 1(5), pp. 1-9. DOI: 10.1038/ncomms1053.

Zhang, P., Sun, H., Ren, C., Min, L. y Zhang, H. (2018). «Sorption mechanisms of neonicotinoids on biochars and the impact of deashing treatments on biochar structure and neonicotinoids sorption». Environmental Pollution, 234, pp. 812-820. DOI: 10.1016/j. envpol.2017.12.013.

Zhang, X. y Xu, J. (2018). «Optimal policies for climate change: A joint consideration of $\mathrm{CO}_{2}$ and methane». Applied Energy, 211, pp. 10211029. DOI: 10.1016/j.apenergy.2017.10.067.

Zimmerman, A. R. (2010). «Abiotic and microbial oxidation of laboratory-produced black carbon (biochar)». Environmental Science \& Technology, 44(4), pp. 1295-1301. DOI: 10.1021/es903140c.

Zomeren, M., Pauls, I. L. y Cohen-Chen, S. (2019). «ls hope good for motivating collective action in the context of climate change? Differentiating hope's emotion- and problem-focused coping functions». Clobal Environmental Change, 58. DOI: 10.1016/j. gloenvcha.2019.04.003. 